\title{
Quality of life and body image after bariatric surgery
}

\begin{abstract}
Obesity is a major public health concerns obesity can cause severe diseases obesity can cause many chronic disease such as diabetes ,heart disease , high blood pressure ,cancer, arthritis, fatty liver and depression related to low self esteem and negative body image. Bariatric surgical procedure had a result in weight loss studies have shown durable weight loss and lipids and improve self-stem and body image. Do you remember When was the last time you looked in the mirror and really appreciated yourself for many people who struggle with obesity this may be unfamiliar experience, Weight loss following bariatric surgery associated with significant improvement in obesity related comorbidities, body satisfaction and psychosocial outcomes.
\end{abstract}

People obviously choose to have bariatric surgery for many reasons

1. Health concerns $62 \%$

2. Improve self-esteem $19 \%$

3. Increase physical functioning $11 \%$

4. Increase social attractiveness $3 \%$

5. Business job attractiveness

Keywords: bariatric surgery, body image, obesity
Volume 8 Issue I - 2018

Sara Shahda

Clinical Nutrition \&Dietitian, Saudi Arabia

Correspondence: Sara Shahda, Clinical Nutrition \&Dietitian, Badana clinic, University of Sharjah, Saudi Arabia, Emailsara_shahda@hotmail.com

Received: December 29, 2017 | Published: January 16, 2018

\section{Body image}

It is psychological factor person perception of their physical appearance or internal sense of how one's body appears to other.

a. It is common to struggle with body image, no matter who you are.

b. Severe negative body image can lead to serious eating and exercise disorders.

c. Feeling good about yourself can affect your mental health and how you behave.

We all have feelings about the way we look your overall body image can range from very positive to very negative. Positive Body image means that most of the time, you see yourself accurately, you feel comfortable in your body, and you feel good about the way you look. Negative Body image develops when someone feels her or his body does not measure up to family, social, or media ideals. Having a negative body image can have a harmful effect on one's health and well-being, and you may want to talk with a professional counselor (Figure 1) (Figure 2).

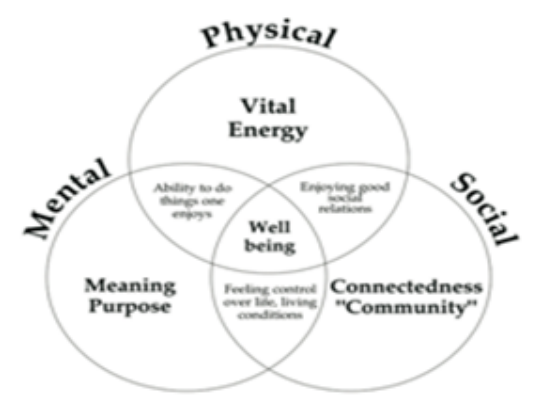

Figure I People obviously choose to have bariatric surgery for many reasons.

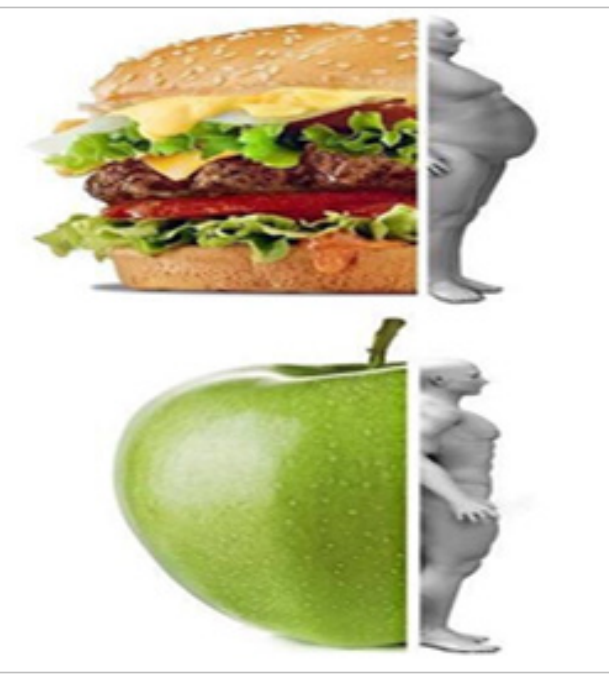

Figure 2 Body image after bariatric Surgery.

\section{What influence body image?}

The people around us and our culture strongly influence it. We get both positive and negative messages we also get messages about body image from television, magazines, films, and other media.

Many of the beliefs we have about the way women and men "should" look coming from the models and celebrities we see in the media.

\section{How can a negative body image affect my health and well-being?}

Having a long negative body image can affect both your mental and physical health. 
I. Have anxiety, depression, and low self-esteem.

II. Cut themselves off from being with other people socially

III. Stop doing healthy activities that require them to show their bodies, such as exercising, or swimming

Suffer from serious mental health problems, such as anorexia, bulimia or overeating. These disorders can be very serious.

\section{Bariatric surgery -how to accept your new life and body}

After Bariatric surgery patient can experience some changes in their eating exercise habits changing in body image, social interests all these can offer special big change, risk and opportunities.

\section{Tips to accept your new life after bariatric surgery?}

a. Remember that health and appearance are two different things.

b. Key is to have realistic expectations about how much changing your appearance can change how you feel about yourself.

c. Accept and value your genes - you probably inherited a lot of traits from your family members, so love those traits as you love your family.

d. Keep a list of your positive qualities that have nothing to do with your appearance.

e. Surround yourself with people who are supportive and who make you feel good about yourself.

f. Treat your body with respect and kindness (Figure 3).

g. People may choose to change their appearance in many ways, for a variety of reasons.

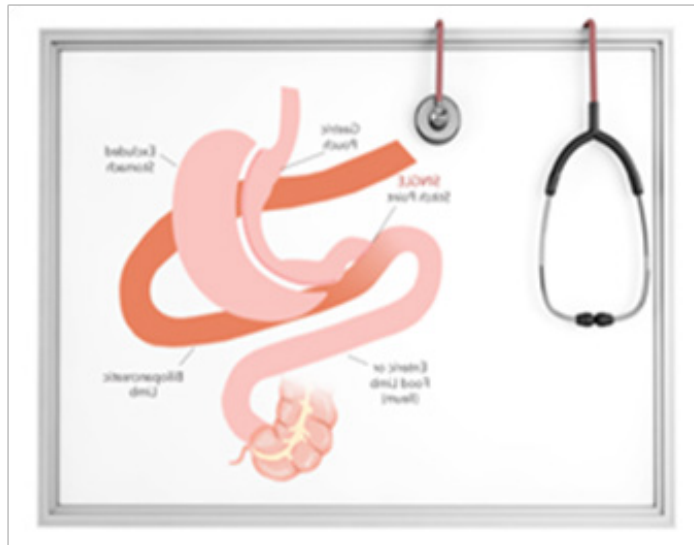

Figure 3 Tips to accept your new life after bariatric surgery.

\section{After years of struggling with obesity bariatric surgery brings big changes effect in quality of life?}

I. Increase Body image satisfaction

II. Enhanced self-Image and self esteem

III. Improvement in psychological and mental health well being

IV. Stability in mood

V. Improved social acceptance

VI. Improvement in social and cultural activities (Figure 4) ${ }^{1-3}$

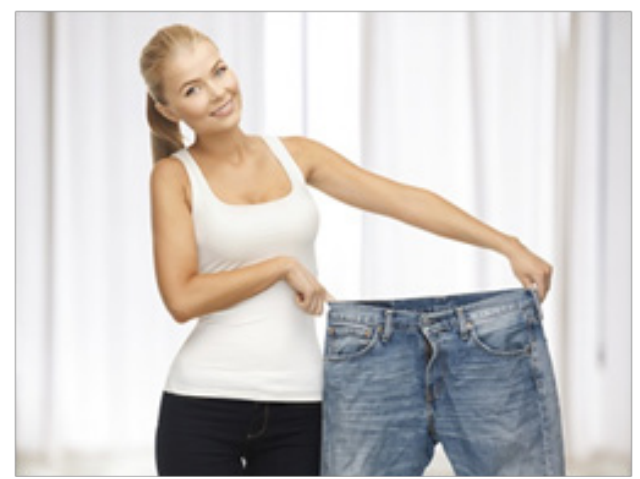

Figure 4 After years of struggling with obesity bariatric surgery brings big changes effect in quality of life.

\section{Conclusion}

We have demonstrated that body image improve after surgical weight loss .healthy body confidence can help you enrich your life, discover new hobbies and live life without worry about your shape in mirror.

\section{Acknowledgements}

None.

\section{Conflict of interest}

The author declares no conflict of interest.

\section{References}

1. Health and Wellness.

2. David Engstrom. Body Image vs. Health Risk: What's Your Motivation for Weight-loss Surgery? 2018.

3. Learn to Love Your Body after Bariatric Surgery. 